\title{
Reliability and Validity of the Hypoxico Everest Summit II Altitude Generator
}

3

4

5

6

8

9

10

11

12

Harwood, B. ${ }^{1 凶}$, Wright, J. ${ }^{1}$, Burnet, S. ${ }^{1}$

${ }^{1}$ School of Sport, Health and Social Sciences, Solent University, Southampton, Hampshire, United Kingdom

$\bowtie$ Ben.Harwood2@outlook.com

Corresponding Author's Address: 11 Westland Road, Yeovil, Somerset. BA20 2AS.

Word Count: 3663 
The Hypoxico Everest Summit II Altitude Generator produces normobaric hypoxic conditions, which are commonly used in altitude acclimation. Despite being used routinely within applied sport science, this system is yet to be independently validated. Thus, the aim of this study was to explore within- and between-day inter- and intramachine reliability and validity. Two Hypoxico Everest Summit II Altitude Generators were investigated for six altitude settings in a randomised order $(0.5,3.0,5.5,8.0$, 10.5, 12.0). Following institutional ethics approval, Hypoxico generated air was sequentially pumped into three Douglas bags, each with a 1-min collection period. Samples were collected on eight occasions, measuring inter- and intra-machine reliability, both within-day (9AM - 2PM) and between-day (48-h) with samples analysed to determine $\mathrm{FiO}_{2}(\%)$ and volume $\left(\mathrm{L} \cdot \mathrm{min}^{-1}\right)$. Coefficient of variation $(\mathrm{CV})$ was calculated for $\mathrm{FiO}_{2}$ and volume for each altitude setting. Mean $\mathrm{CV}$ for $\mathrm{FiO}_{2}$ did not exceed $0.42 \%$ within-day, $0.49 \%$ between-day, and $0.81 \%$ inter-machine. Volume mean CV did not exceed $0.85 \%$ within-day, $0.91 \%$ between-day, and $1.17 \%$ intermachine. One-sampled t-tests were conducted comparing Hypoxico reference values with collected samples using equivalent $\mathrm{FiO}_{2}$ for each altitude setting with a Bonferroni corrected significance set at $P<0.004$. Settings 3.0, 5.5, 8.0, 10.5, and 12.0 all produced significantly different $(P<0.001) \mathrm{FiO}_{2}$ concentrations than the reference values stated $(-0.51,-0.97,-0.72,+0.41$, and $+0.40 \%$, respectively). Altitude setting and 1-min volume had an inverse relationship. All settings produced a significantly lower $(P<0.001)$ volume by 21.80 to $35.40 \mathrm{~L} \cdot \mathrm{min}^{-1}$ as compared to the company's claim of $126.6 \mathrm{~L} \cdot \mathrm{min}^{-1}$. The Hypoxico Everest Summit II produced a reliable and consistent air feed; however, the manufacturer's reference values demonstrated poor agreement with machine produced $\mathrm{FiO}_{2}$ and volume. Therefore, it is recommended that athletes, practitioners and researchers self-validate the altitude generator postwarm-up using an accurate oxygen sensor for accuracy and safety purposes.

Keywords: Normobaric, Hypoxia, Altitude Simulator, Altitude Training, Hypoxic, Hypoxico 


\section{Introduction}

The recent rise in the popularity of extreme sports tourism and altitude training has resulted in an increased demand for altitude simulation devices. These devices create normobaric hypoxia, where hypoxic air is produced through a decrease in the fraction of inspired oxygen $\left(\mathrm{FiO}_{2}\right) .{ }^{[1]}$ This method of hypoxia production offers a more logistically and economically viable approach to altitude training and acclimatisation as opposed to its hypobaric alternative. A variety of normobaric hypoxia altitude machines are now available to the general public for purchase.

One publicly available altitude generator that produces normobaric hypoxia is the Hypoxico Everest Summit II. This machine is designed to allow breath-by-breath exposure, intermittent hypoxic training and hypoxia for altitude chambers/tents. The device offers 24 altitude settings ranging from $0.5-12.0$ with a claimed peak altitude of $3962 \mathrm{~m}$ at the setting of $12.0^{.{ }^{[2]}}$ The settings represent altitude increments of various magnitude, and to the authors' knowledge do not represent any specific geographical altitude training locations. The manufacturers also claim the machine to have an airflow of $7600 \mathrm{~L} \cdot \mathrm{h}^{-1}$, equating to an equivalent $126.6 \mathrm{~L} \cdot \mathrm{min}^{-1} .{ }^{[3]}$

The Hypoxico Everest Summit II is an altitude simulator that has been used within research to produce hypoxic conditions. ${ }^{[4-7]}$ Spurling, Zammit and Lozewicz ${ }^{[4]}$ employed the Hypoxico to produce normobaric hypoxia in the evaluation of at-risk patients during air travel. The product is also available for public consumption with Hypoxico's wider clientele including Olympic committees, universities, and the US army ${ }^{[8]}$ Despite the machine's use in research and its public availability, the Hypoxico has yet to be deemed reliable and independently validated from empirical research. Moreover, the reference values provided by Hypoxico for both $\mathrm{FiO}_{2}$ and volume are unvalidated.

To the authors' knowledge, the only current independent data regarding the Hypoxico is provided by the Spurling, Zammit and Lozewicz study, ${ }^{[4]}$ stating at an unspecified altitude setting, the Hypoxico demonstrated a constant output of $15 \pm 0.1 \% \mathrm{FiO}_{2}$ for more than 1 hour. This data demonstrates consistency in output, but provides no testretest measures or specific reference-value testing. Whilst it is acknowledged that the machine's output is likely to be monitored by academic institutions for research and 
114 training, the general public is unlikely to monitor hypoxic conditions. The plethora of risks associated with hypoxia are addressed by Hull, ${ }^{[9]}$ who states excessive exposure can induce altitude sickness, and in serious cases, cause pulmonary or cerebral oedemas. Although rare, the likelihood of these risks is increased without prior authentication of the available system, and small variations in $\mathrm{FiO}_{2}$ may make the difference between safe and dangerous normobaric hypoxic conditions. Validation of the system's functionalities and manufacturer's claims will provide unbiased objective evidence for use by both practitioners and the general public.

Therefore, the aim of this study was to explore the reliability and validity of the Hypoxico Everest Summit II Altitude Generator. This research set three objectives: explore within- and between-day inter- and intra-machine reliability, examine the validity of machine-provided reference values, and validate the altitude simulator for personal and research utilisation.

.




\section{Method}

\subsection{Study Design}

A correlational test-retest study design was employed investigating two Hypoxico Everest Summit II Altitude Generators. Machine number, time of testing and day of testing were selected and $\mathrm{FiO}_{2}(\%)$ and volume $\left(\mathrm{L} \cdot \mathrm{min}^{-1}\right)$ produced by the Hypoxico were measured. Equivalent $\mathrm{FiO}_{2}$ values for each altitude setting were attained from the Hypoxico altitude to equivalent oxygen calculator, ${ }^{[10]}$ with calculated concentrations used as the reference values for the validity component of the study. The six tested altitude settings corresponded to the following $\mathrm{FiO}_{2}$ values; $0.5: 20.5 \%$, 3.0: $18.8 \%, 5.5$ : $17.0 \%$, 8.0: 14.9\%, 10.5: 12.9\%, 12.0: $12.7 \%$.

\subsection{Data Collection: Protocol}

Prior to commencing the study, ethical approval was granted by the host University's Ethics Committee. Two Hypoxico Everest Summit II Altitude Generators (The Altitude Centre, UK) were tested over six altitude settings $(0.5,3.0,5.5,8.0,10.5,12.0)$. The settings refer to an equivalent altitude of $162,884,1707,2761,3886$, and $3962 \mathrm{~m}$, respectively. These settings were chosen to allow the investigation of reliability and validity at a multitude of points over the range of hypoxic intensities produced by the generator at altitudes similar to those commonly utilised for acclimatisation and altitude training purposes. Data were collected from each machine over the tested settings on four separate occasions. These samples were taken within-day (9AM and 2PM) and between-day (48-h) with the protocol shown in Fig. 1. The order of altitude settings sampled was randomised every session using the WINPEPI Software. ${ }^{[11]}$

\section{*INSERT FIGURE 1 HERE*}

Figure 1. Study protocol demonstrating the manner in which within-day, between-day, and between-machine comparisons were made. The randomised order of altitude settings tested is presented in brackets next to each testing period.

Prior to each data collection session, atmospheric conditions, such as temperature $\left({ }^{\circ} \mathrm{C}\right)$ and atmospheric pressure $(\mathrm{mmHg})$, were noted to ensure the standardisation of measurements. All testing was completed in a laboratory that was at an altitude of 12 
$\mathrm{m}$, thus minimising the effect of a lower ambient barometric pressure on machine $\mathrm{FiO}_{2}$. Furthermore, temperature was measured to ensure temperature's influence on volume could be accounted for as per Charles' Law.

Firstly, the Gas Analysis System (1440D, Servomex, UK) was calibrated according to the operator's guidelines. ${ }^{[12]}$ The system was initially baseline zeroed using a 99.9\% $\mathrm{N}$ solution (BOC Gases Ltd, UK). Subsequently, a further solution containing $15 \% \mathrm{O}_{2}$, $5 \% \mathrm{CO}_{2}$ and $80 \% \mathrm{~N}$ (BOC Gases Ltd, UK) was used to calibrate mid-level readings. Finally, ambient air was pumped through the system to verify the calibration procedure. This process was completed prior to the beginning of each data collection session, and after three altitude settings had been tested within a session. Preceding data collection, the Douglas bag was also thoroughly checked to ensure collection bags remained sealed and stopcocks were fully functional. The system was manually attached using adhesive and checked using a Dry Gas Meter (Harvard Apparatus, UK) to confirm a complete seal prior to each data collection session.

Whilst calibration and equipment checks occurred, the Hypoxico underwent a 10-min warm-up at the chosen altitude setting as suggested by Hypoxico. ${ }^{[2]}$ Following the warm-up, Hypoxico generated air was sequentially pumped into three Douglas bags, each with a 1-min collection period manually timed via a stopwatch. The duration (s) of sample collection was noted for each bag to ensure volume could be corrected and accurately compared against Hypoxico's claim of a $126.6 \mathrm{~L} \cdot \mathrm{min}^{-1}$ airflow. ${ }^{[3]}$ The bags' contents were individually analysed using the gas analyser, from which $\mathrm{FiO}_{2}(\%)$ was calculated. Following this, the generated air was vacuumed using the Air Evacuation Unit. Simultaneous measurement of each bag's evacuated volume $\left(L \cdot \mathrm{min}^{-1}\right)$ and temperature $\left({ }^{\circ} \mathrm{C}\right)$ occurred via the Harvard Dry Gas Meter.

\subsection{Statistical Analysis}

Statistical analysis was conducted using Microsoft Excel 365 (Microsoft, USA) and SPSS, version 24 (IBM, Germany). Descriptive data was presented as mean \pm standard deviation (SD). Coefficient of variation (CV) was calculated for each altitude setting within- and between-day, for inter- and intra-machine. One-sampled t-tests were used to compare collected $\mathrm{FiO}_{2}$ to Hypoxico's claimed reference values at each altitude setting, as well as the volume compared to Hypoxico's claim of a $126.6 \mathrm{~L} \cdot \mathrm{min}^{-}$ 
$201{ }^{1}$ output. ${ }^{[3]}$ Due to conducting multiple analyses on this data set, the Bonferroni 202 correction was employed with statistical significance set at $P<0.004$. ${ }^{[13]}$

203

204

205

206

207

208

209

210

211

212

213

214

215

216

217

218

219

220

221

222

223

224

225 


\section{Results}

227 Mean atmospheric pressure during data collection was $762 \pm 6 \mathrm{mmHg}$, mean room 228 temperature was $17.5 \pm 0.6^{\circ} \mathrm{C}$, and mean bag collection time was $59.89 \pm 0.11 \mathrm{~s}$.

\section{3.1. Reliability Results}

230 Table 1 demonstrates machine 1 and 2's respective CV at varying altitude settings for 231 intra-machine within- and between-day reliability. Table 1 also provides a between232 day, inter-machine $\mathrm{CV}$, utilising all samples collected throughout testing. 
Table 1. Machine 1 \& 2 coefficient of variation data

\begin{tabular}{|c|c|c|c|c|c|c|c|c|c|c|c|c|c|c|}
\hline \multirow[t]{2}{*}{$\begin{array}{l}\text { Altitude } \\
\text { Setting }\end{array}$} & \multicolumn{2}{|c|}{$\begin{array}{c}\text { Within-Day } \\
\text { Day } 1 \\
\text { Machine 1 } \\
\end{array}$} & \multicolumn{2}{|c|}{$\begin{array}{c}\text { Within-Day } \\
\text { Day } 2 \\
\text { Machine } 1\end{array}$} & $\frac{\text { Mac }}{C V}$ & $\frac{\text { hine } 1}{(\%)}$ & \multicolumn{2}{|c|}{$\frac{\text { Machine } 2}{\text { CV (\%) }}$} & \multicolumn{2}{|c|}{$\frac{\text { Machine } 2}{\text { CV (\%) }}$} & \multicolumn{2}{|c|}{$\frac{\text { Machine 2 }}{\text { CV (\%) }}$} & \multicolumn{2}{|c|}{$\frac{\text { Machine } 1 \& 2}{\text { CV (\%) }}$} \\
\hline & $\mathrm{FiO}_{2}$ & Volume & $\mathrm{FiO}_{2}$ & Volume & $\mathrm{FiO}_{2}$ & Volume & $\mathrm{FiO}_{2}$ & Volume & $\mathrm{FiO}_{2}$ & Volume & $\mathrm{FiO}_{2}$ & Volume & $\mathrm{FiO}_{2}$ & Volume \\
\hline 0.5 & 0.27 & 0.49 & 0.00 & 0.52 & 0.22 & 0.57 & 0.27 & 0.27 & 0.00 & 1.15 & 0.22 & 0.92 & 0.54 & 1.14 \\
\hline 3.0 & 0.30 & 1.06 & 0.28 & 0.45 & 0.28 & 1.01 & 0.22 & 0.39 & 0.30 & 0.58 & 0.31 & 0.63 & 0.78 & 1.05 \\
\hline 5.5 & 0.00 & 0.19 & 0.32 & 0.26 & 0.31 & 0.42 & 0.34 & 0.38 & 0.93 & 1.26 & 0.97 & 0.93 & 1.06 & 1.38 \\
\hline 8.0 & 0.00 & 0.50 & 0.37 & 1.10 & 0.35 & 0.81 & 0.00 & 1.06 & 0.36 & 0.26 & 0.27 & 0.83 & 1.07 & 0.97 \\
\hline 10.5 & 0.39 & 0.80 & 0.00 & 0.59 & 0.37 & 0.85 & 0.82 & 1.62 & 0.31 & 0.91 & 0.67 & 1.15 & 0.64 & 1.33 \\
\hline 12.0 & 0.83 & 0.74 & 0.00 & 0.59 & 0.98 & 1.27 & 0.31 & 0.53 & 0.62 & 0.91 & 0.51 & 0.98 & 0.76 & 1.17 \\
\hline $\begin{array}{l}\text { Mean } \pm \\
\text { SD }\end{array}$ & $\begin{array}{c}0.30 \pm \\
0.31\end{array}$ & $\begin{array}{c}0.63 \pm \\
0.30\end{array}$ & $\begin{array}{c}0.16 \pm \\
0.18\end{array}$ & $\begin{array}{c}0.58 \pm \\
0.28\end{array}$ & $\begin{array}{c}0.42 \pm \\
0.28\end{array}$ & $\begin{array}{c}0.82 \pm \\
0.31\end{array}$ & $\begin{array}{c}0.33 \pm \\
0.27\end{array}$ & $\begin{array}{c}0.71 \pm \\
0.52\end{array}$ & $\begin{array}{c}0.42 \pm \\
0.32\end{array}$ & $\begin{array}{c}0.85 \pm \\
0.37\end{array}$ & $\begin{array}{l}0.49 \pm \\
0.29\end{array}$ & $\begin{array}{c}0.91 \pm \\
0.17\end{array}$ & $\begin{array}{c}0.81 \pm \\
0.22\end{array}$ & $\begin{array}{c}1.17 \pm \\
0.16\end{array}$ \\
\hline
\end{tabular}




\subsection{Validity Results}

Following the low level of variance in $\mathrm{FiO}_{2}$ and volume found within each altitude setting, the validity component of this research was conducted by combining the data from the two machines on the four collection dates. A Bland-Altman plot was used to compare the altitude settings as shown below in Fig. 2, where reference values overestimated produced $\mathrm{FiO}_{2}$ in mid-range altitude settings, yet underestimated $\mathrm{FiO}_{2}$ in higher altitude settings.

\section{*INSERT FIGURE 2 HERE*}

Figure 2. Bland-Altman plot of the limits of agreement between the Hypoxico collected samples and the Hypoxico reference values. The solid black line represents the mean difference in $\mathrm{FiO}_{2}$ and the dashed lines represent the upper and lower 95\% limits of agreement.

Results of a one-sampled t-test demonstrate several altitude settings had a significantly different $\mathrm{FiO}_{2}$ compared to their corresponding reference values with full details shown below in Table 2. Setting 0.5 had a non-significant $(P=0.283)$ mean difference of $-0.02 \%$. Setting 3.0 had a significant $(P<0.001)$ mean difference of $0.51 \%$. Setting 5.5 had a significant $(P<0.001)$ mean difference of $-0.97 \%$. Setting 8.0 had a significant $(P<0.001)$ mean difference of $-0.72 \%$. Setting 10.5 had a significant $(P<0.001)$ mean difference of $+0.41 \%$ and setting 12.0 had a significant $(P<0.001)$ mean difference of $+0.40 \%$.

Table 2. $\mathrm{FiO}_{2}$ reference value one-sampled $t$-tests

\begin{tabular}{ccccc}
\hline $\begin{array}{c}\text { Altitude } \\
\text { Setting }\end{array}$ & $\begin{array}{c}\text { Mean } \mathrm{FiO}_{2} \\
(\%)\end{array}$ & $\begin{array}{c}\text { Machine } \\
\text { Reference } \\
\mathrm{FiO}_{2}(\%)\end{array}$ & One-Sampled t-test & $\begin{array}{c}\text { Reference Value } \\
\text { Difference (95\% Cl) } \\
(\%)\end{array}$ \\
\hline 0.5 & $20.48 \pm 0.11$ & 20.5 & $t_{(23)}=-1.100, P=0.283$ & $-0.02(-0.07,0.02)$ \\
3.0 & $18.29 \pm 0.14$ & 18.8 & $t_{(23)}=-17.634, P<0.001^{*}$ & $-0.51(-0.57,-0.45)$ \\
5.5 & $16.03 \pm 0.17$ & 17.0 & $t_{(23)}=-27.875, P<0.001^{*}$ & $-0.97(-1.04,-0.90)$ \\
8.0 & $14.18 \pm 0.15$ & 14.9 & $t_{(23)}=-23.509, P<0.001^{*}$ & $-0.72(-0.79,-0.66)$ \\
10.5 & $13.31 \pm 0.09$ & 12.9 & $t_{(23)}=23.769, P<0.001^{*}$ & $+0.41(0.38,0.45)$ \\
12.0 & $13.10 \pm 0.10$ & 12.7 & $t_{(23)}=19.818, P<0.001^{*}$ & $+0.40(0.36,0.45)$ \\
\hline
\end{tabular}


A low level of variance in measured volume was seen within each altitude setting; however, produced volume linearly decreased with an increase in altitude. This occurred despite the Hypoxico claimed volume of $126.6 \mathrm{~L} \cdot \mathrm{min}^{-1}{ }^{1}{ }^{[3]}$ The average 1 -min volume produced by the Hypoxico at an altitude setting of 0.5 was $104.80 \pm 1.20 \mathrm{~L} \cdot \mathrm{min}$ ${ }^{1}$, whereas at an altitude setting of 12.0, only $91.20 \pm 1.07 \mathrm{~L} \cdot \mathrm{min}^{-1}$ was produced (Fig. $3)$.

\section{*INSERT FIGURE 3 HERE*}

Figure 3. Hypoxico mean 1-min volume at altitude settings of 0.5, 3.0, 5.5, 8.0, 10.5 and 12.0 with a linear regression line (solid black line). Error bars represent $\pm 1 S D$.

One-sampled t-tests were conducted using the samples collected at each altitude setting. These were compared against the claimed volume of $126.6 \mathrm{~L} \cdot \mathrm{min}^{-1}$, with full results displayed in Table 3. Setting 0.5 had a significant $(P<0.001)$ mean difference of $-21.80 \mathrm{~L} \cdot \mathrm{min}^{-1}$. Setting 3.0 had a significant $(P<0.001)$ mean difference of -24.25 $\mathrm{L} \cdot \mathrm{min}^{-1}$. Setting 5.5 had a significant $(P<0.001)$ mean difference of $-26.55 \mathrm{~L} \cdot \mathrm{min}^{-1}$. Setting 8.0 had a significant $(P<0.001)$ mean difference of $-29.74 \mathrm{~L} \cdot \mathrm{min}^{-1}$. Setting 10.5 had a significant $(P<0.001)$ mean difference of $-33.14 \mathrm{~L} \cdot \mathrm{min}^{-1}$ and setting 12.0 had a significant $(P<0.001)$ mean difference of $-35.40 \mathrm{~L} \cdot \mathrm{min}^{-1}$.

Table 3. Volume reference value one-sampled t-tests

\begin{tabular}{cccc}
\hline $\begin{array}{c}\text { Altitude } \\
\text { Setting }\end{array}$ & $\begin{array}{c}\text { Mean } \\
\text { Volume } \\
\left(\mathbf{L} \cdot \mathbf{m i n}^{-1}\right)\end{array}$ & One-Sampled t-test & $\begin{array}{c}\text { Reference Value } \\
\text { Difference }(95 \% \text { Cl) } \\
\left(\mathbf{L} \cdot \mathbf{m i n}^{-1}\right)\end{array}$ \\
\hline 0.5 & $104.80 \pm 1.20$ & $t_{(23)}=-89.049, P<0.001^{*}$ & $-21.80(-22.30,-21.29)$ \\
3.0 & $102.35 \pm 1.07$ & $t_{(23)}=-110.991, P<0.001^{*}$ & $-24.25(-24.71,-23.80)$ \\
5.5 & $100.05 \pm 1.38$ & $t_{(23)}=-94.355, P<0.001^{*}$ & $-26.55(-27.14,-25.97)$ \\
8.0 & $96.86 \pm 0.94$ & $t_{(23)}=-155.404, P<0.001^{*}$ & $-29.74(-30.14,-29.35)$ \\
10.5 & $93.46 \pm 1.24$ & $t_{(23)}=-130.530, P<0.001^{*}$ & $-33.14(-33.66,-32.61)$ \\
12.0 & $91.20 \pm 1.07$ & $t_{(23)}=-162.354, P<0.001^{*}$ & $-35.40(-35.86,-34.95)$ \\
\hline
\end{tabular}

${ }^{*}$ denotes a statistically significant Bonferroni corrected $P$ value $(<0.004)$ 


\section{Discussion}

Firstly, this research set the objective of exploring within- and between-day inter- and intra-machine reliability. The machine's production of $\mathrm{FiO}_{2}$ at each altitude setting was consistent, with Table 1 showing an overall average CV of $<1 \%$. Mean CV ranged from $0.00-0.93 \%$ amongst settings within-day, $0.22-0.98 \%$ between-day, and 0.54 $-1.07 \%$ inter-machine. Due to the design of this study, a true within-day inter-machine comparison was not conducted and, therefore, between-day differences may provide a confounding variable in inter-machine comparisons and reliability. Regardless, intermachine CV remained low. With an average CV value of $0.81 \pm 0.22 \%$ and no individual setting $\mathrm{CV}$ exceeding $1.07 \%$ in $\mathrm{FiO}_{2}$, an excellent level of consistency and reliability was demonstrated. The increase in between-day CV is likely a result of acute alterations within the ambient environment, such as atmospheric pressure. Furthermore, the marginally larger difference in inter-machine comparisons may be accounted for by the between-day confounding variable, and small variations in manufactured hypoxia-producing components, such as the pressure swing adsorption chamber and carbon molecular sieve.

Moreover, the volume of the air produced by the Hypoxico was also consistent. An average $\mathrm{CV}$ of $<1.2 \%$ was found throughout all testing. Mean $\mathrm{CV}$ ranged from 0.19 $1.62 \%$ amongst settings within-day, $0.42-1.27 \%$ between-day, and $0.97-1.38 \%$ inter-machine. The minimal variation throughout demonstrates a steady, consistent production of normobaric hypoxia.

The second objective of this research was to examine the validity of the machineprovided reference values. As shown in Fig. 2, acceptable agreement was seen in setting 0.5 , but within mid-range settings a higher hypoxic intensity than the corresponding reference values was produced. However, this shifted to a lower hypoxic intensity in comparison to reference values within higher altitude settings. The $\mathrm{FiO}_{2}$ collected at settings $3.0,5.5$, and 8.0 were significantly lower $(P<0.001)$ than the Hypoxico reference values by $0.51 \%, 0.97 \%$, and $0.72 \%$, respectively. Conversely, $\mathrm{FiO}_{2}$ collected at settings 10.5 and 12.0 were significantly higher $(P<0.001)$ than the Hypoxico reference values $(0.41 \%$ and $0.40 \%$, respectively). This shift in agreement 
may be a consequence of the altitude generator attaining a maximum output, stunting the expected increase in hypoxic intensity within higher altitude settings.

The largest disagreement between collected and reference $\mathrm{FiO}_{2}$ values was seen in settings 5.5 and 8.0 with differences of $-0.97 \%$ and $-0.72 \%$, equating to an equivalent increase in altitude of approximately 473 and $389 \mathrm{~m}$, respectively. Assuming this midsetting overestimation of $\mathrm{FiO}_{2}$ exists in all machines, a much greater hypoxic stimulus than anticipated may be delivered. However, as this disagreement occurs in the midrange altitude settings, during short periods of use the previously discussed risks of hypoxia are unlikely to be of concern. Nonetheless, this is a large discrepancy to exist in equipment that may be used without supervision for extended lengths of exposure, with Hypoxico recommending use of the machine for $7-9$ hours per night in a livehigh train-low approach. ${ }^{[14]}$ Furthermore, whilst the underestimation in $\mathrm{FiO}_{2}$ at higher altitude settings is not a safety concern, a lower hypoxic intensity than anticipated may diminish the effectiveness of acclimation and altitude training programmes.

The exact cause of the extent of disagreement within the moderate altitude settings is unknown, although this may possibly arise from the pressure swing adsorption method of hypoxia production. Within settings $3.0-8.0$, the greater hypoxic intensity is likely caused by an excess in chamber pressure, resulting in greater carbon filtration and consequently a lower produced $\mathrm{FiO}_{2}$. This may be caused by an overestimation in pressure requirements for carbon filtration or a lack of internal calibration. The magnitude of difference between settings may not replicate in setting 0.5 due to the minimal increase in chamber pressure required to produce an $\mathrm{FiO}_{2}$ of $20.5 \%$, limiting the influence of any overestimation in pressure requirements. Furthermore, within settings $10.5-12.0$, it is likely that carbon filtration reaches a near-maximal rate due to a peak pressure being attained, resulting in a plateau in $\mathrm{FiO}_{2}$ and hypoxic intensity. It would appear this plateau has not been accounted for within the Hypoxico altitude settings, consequently explaining the transition from reference values overestimating $\mathrm{FiO}_{2}$ concentration in mid-range settings, to underestimating $\mathrm{FiO}_{2}$ in higher settings.

The local altitude at which the unit is operated may also further impact hypoxic intensity, with air intake at higher altitudes possessing a lower barometric pressure than that found at sea-level. ${ }^{[1]}$ Whilst this study was completed at a local altitude of 12 $\mathrm{m}$, machine use at higher altitudes may result in a greater overestimation in the 
pressure requirements for carbon filtration, reducing produced $\mathrm{FiO}_{2}$ and likely decreasing machine volume output. To the authors' knowledge, a supplementary table to help account for adjusted machine output in different altitudes is not currently supplied, and thus the use of an $\mathrm{O}_{2}$ concentration device prior to hypoxic exposure is recommended to accurately monitor $\mathrm{FiO}_{2}$.

In relation to volume, Table 3 demonstrates that each of the altitude settings produced a significantly lower value $(P<0.001)$ than the Hypoxico claim of $126.6 \mathrm{~L} \cdot \mathrm{min}^{-1}$.[3] The mean volume ranged from $21.80-35.40 \mathrm{~L} \cdot \mathrm{min}^{-1}$ lower than the reference value. Data collection commenced directly after the Hypoxico instructed 10-min warm-up and subsequently, a longer warm-up period may be required to increase volume output. Furthermore, due to the limitation of a small Douglas bag capacity, this comparison was made by dividing the 1-h Hypoxico claim to provide a value for 1-min. Assuming a constant output when compared to the $7600 \mathrm{~L} \cdot \mathrm{h}^{-1}$ claim, amongst settings this 1 -min difference amounts to a $\sim 1308-2124 \mathrm{~L} \cdot \mathrm{h}^{-1}$ shortfall in volume. However, a limitation of this study design is any $\mathrm{FiO}_{2}$ or volume fluctuation following the first 3-min of collection would have gone unnoticed. A large difference in volume output was also seen between altitude settings. Setting 0.5 produced a mean volume of $104.80 \pm 1.20$ $\mathrm{L} \cdot \mathrm{min}^{-1}$, while setting 12.0 produced a mean volume of $91.20 \pm 1.07 \mathrm{~L} \cdot \mathrm{min}^{-1}$, indicative of an inverse relationship. This is assumed to be a consequence of greater oxygen retention via the altitude generator's molecular sieve technology at higher altitude settings to decrease $\mathrm{FiO}_{2}$. Assuming a constant external air feed, output volume would expectedly lower with an increase in altitude setting.

These findings have different consequences depending on the modality of machine use. Whilst a lower volume than stated may not implicate hypoxic exposure in moderate breath-by-breath use, during high intensity exercise minute ventilation can exceed >100 L.min ${ }^{-1}{ }^{[15]}$ Consequently, breath-by-breath exposure at high workloads may elicit minute ventilations that exceed the rate of the altitude generator's hypoxic air production and thus, possibly flaw the hypoxic stimulus. This may also be further exacerbated by the acute hyperventilation that typically occurs in response to hypoxic exposure. ${ }^{[16]}$ Insufficient volume may also disrupt hypoxic supply to an altitude tent system; however, a minimal output of $90 \mathrm{~L} \cdot \mathrm{min}^{-1}$ is likely sufficient to maintain an appropriate volume production in a moderately sized altitude tent during rest or light 
exercise. Conversely, disagreement in $\mathrm{FiO}_{2}$ output is likely to be greater in an altitude tent system in comparison to breath-by-breath use. The design of the Hypoxico altitude mask enables an immediate evacuation of expired air via a one-way valve, ensuring a constant supply of fresh hypoxic air, an unviable feature in the altitude tent. The large tent volume and method of hypoxic delivery in an altitude tent results in the hypoxic stimulus being a product of both generated air and previously expired gases containing a naturally lower $\mathrm{FiO}_{2}$ concentration. As such, greater variation in $\mathrm{FiO}_{2}$ output may also be present during altitude tent use, with the magnitude of disagreement in $\mathrm{FiO}_{2}$ values likely to be dependent on many factors, including the tent size, ventilation rate, hypoxic volume production rate and hypoxic intensity utilised.

From a practical perspective, the altitude generator is reliable, but the reference values lack validity in both $\mathrm{FiO}_{2}$ and volume. As discussed, a lower volume output and mid to high-range significant differences in $\mathrm{FiO}_{2}$ production may impact the machine's practical function. Therefore, the applications of this research are as follows; researchers, practitioners and general consumers should purchase or ensure the use of an accurate oxygen concentration measurement device prior to hypoxic exposure. The $\mathrm{FiO}_{2}$ concentration should be measured following the warm-up period prior to use to avoid any fluctuations. Based on the results of this study, after an altitude setting is tested once, users can be confident of a reliable future output. Finally, the reliability of the altitude generator should be measured over an extended period and in line with a typical hypoxic training intervention, as suggested by Wright et al. ${ }^{[17]}$

It is suggested future research investigates the remaining untested reference values to further understand the machine's validity. Should the reference values continue to lack agreement with the produced conditions, they may require amendment. 


\section{Conclusion}

The Hypoxico Everest Summit II Altitude Generator produces stable $\mathrm{FiO}_{2}$ and volume values over a range of altitude settings. However, the machine demonstrates limited validity in comparison to the provided altitudes, particularly in the mid-range settings. Furthermore, assuming a consistent output, produced volume is significantly lower than the manufacturer's claim. It is highly recommended that athletes, practitioners and researchers use an accurate oxygen sensor following the 10-min machine warmup to self-validate the equipment for both accuracy and safety purposes. 


\section{Acknowledgements}

457 The authors thank all those who contributed their time in helping this study come to 458 fruition.

459

460

\section{Funding}

461 This research received no specific grant from any funding agency in the public, 462 commercial, or not-for-profit sectors.

463

464

465

466

\section{Declaration of Conflicting Interests}

The authors declare no potential conflicts of interest with respect to the research, authorship, and/or publication of this article. 


\section{Reference List}

1. Coppel J, Hennis P, Gilbert-Kawai E, et al. The physiological effects of hypobaric hypoxia versus normobaric hypoxia: a systematic review of crossover trials. Extrem Physiol Med 2015; 4:2.

2. Hypoxico. Hypoxico Everest Summit II Manual, https://brandfolder.com/hypoxico/attachments/bvzpflze/hypoxico-altitude-trainingsystems-esii-manual-2012-document.pdf?dl=true (2012, accessed 28 November 2018).

3. Hypoxico. Hypoxico Everest Summit II, https://hypoxico.com/product/everestsummit-ii/ (2016, accessed 29 November 2018).

4. Spurling KJ, Zammit $C$ and Lozewicz S. Mains-powered hypoxic gas generation: a cost-effective and safe method to evaluate patients at risk from hypoxia during air travel. Thorax 2011; 66: 731-732.

5. Czuba M, Fidos-Czuba O, Płoszczyca K, et al. Comparison of the effect of intermittent hypoxic training vs. the live high, train low strategy on aerobic capacity and sports performance in cyclists in normoxia. Biol Sport 2018; 35: 39-48.

6. Horiuchi M, Ni-I-Nou A, Miyazaki M, et al. Impact of Resistance Exercise under Hypoxia on Postexercise Hemodynamics in Healthy Young Males. Int $J$ Hypertension 2018; 1456972-10.

7. Dobashi S, Koyama K, Endo J, et al. Impact of Dietary Nitrate Supplementation on Executive Function During Hypoxic Exercise. High Alt Med Biol 2019; 20: 187-191.

8. Hypoxico. Hypoxico Clientele, https://hypoxico.com/clientele/ (2017, accessed 28 November 2018).

9. Hull FM. Altitude sickness. RCGP 1978; 28: 473-477.

10. Hypoxico. Hypoxico Altitude To Oxygen Chart, https://hypoxico.com/altitude-tooxygen-chart/ (2020, accessed 30 December 2020).

11. Abramson JH. WINPEPI (PEPI-for-Windows): computer programs for epidemiologists. Epidemol Perspect Innov 2011; 8: 1. 
12. Servomex. Servomex 1440D Gas Analyser Manual, https://www.servomex.com/downloads/manuals-and-certificates (2019, accessed 15 March 2019).

13. Armstrong RA. When to use the Bonferroni correction. Opthalmic Physiol Opt 2014; 34: 502-508.

14. Hypoxico. Sleeping at Altitude, https://hypoxico.com/sleeping-at-altitude/ (2016, accessed 05 April 2019).

15. Blackie SP, Fairbarn MS, McElvaney NG, et al. Normal Values and Ranges for Ventilation and Breathing Pattern at Maximal Exercise. Chest 1991; 100: 136-142.

16. Calbet JAL, Lundby C. Air to Muscle O2 Delivery during Exercise at Altitude. High Alt Med \& Biol 2009; 10: 123-134

17. Wright J, Walker T, Burnet S, et al. The Reliability and Validity of the PowerTap P1 Power Pedals Before and After 100 Hours of Use. Int J Sports Physiol Perform 2019; 14: 855-858.

2 
Monday: Machine 1 $\left.\left.\begin{array}{l}\text { 9AM }(12.0,10.5,8.0,5.5,3.0,0.5) \\ \text { 2PM }(12.0,5.5,8.0,0.5,3.0,10.5)\end{array}\right]\right] \begin{aligned} & \text { Within- } \\ & \text { Day } 1\end{aligned}$

Wednesday: Machine 1

9AM $(8.0,10.5,3.0,5.5,0.5,12.0)] 7$ Within2PM $(10.5,0.5,12.0,3.0,5.5,8.0)]$ Day 2

Between-

Day Machine 1

Tuesday: Machine 2

9AM $(10.5,5.5,12.0,8.0,3.0,0.5)] 7$ Within2PM $(8.0,5.5,12.0,3.0,0.5,10.5)]$ Day 1

Thursday: Machine 2

9AM $(5.5,12.0,10.5,3.0,0.5,8.0)] 7$ Within2PM $(0.5,3.0,10.5,12.0,5.5,8.0)]$ Day 2

Between-

Day

Machine 2

Between-

Machine

Figure 1. Study protocol demonstrating the manner in which within-day, between-day, and between-machine comparisons were made. The randomised order of altitude settings tested is presented in brackets next to each testing period.

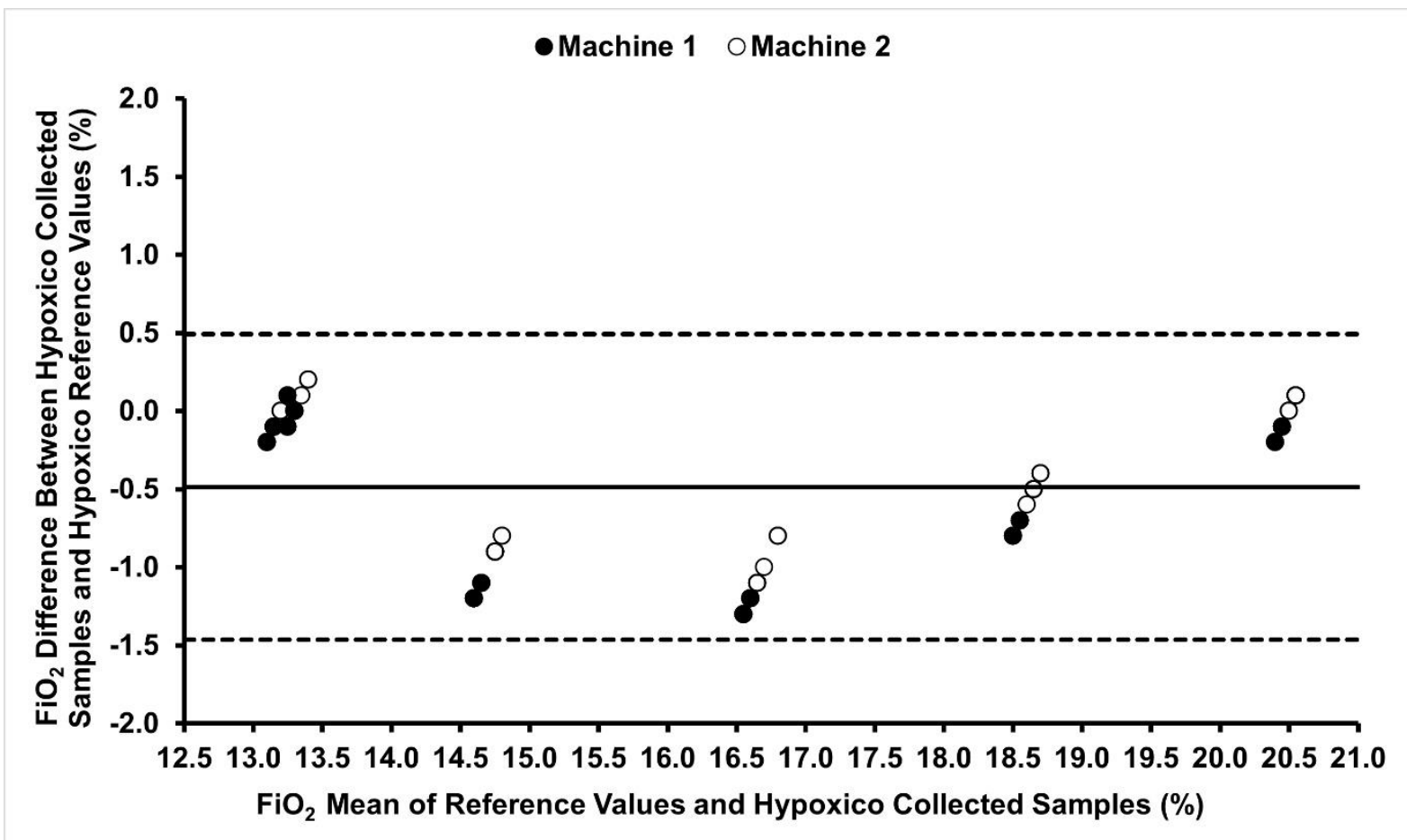

Figure 2. Bland-Altman plot of the limits of agreement between the Hypoxico collected samples and the Hypoxico reference values. The solid black line represents the mean difference in $\mathrm{FiO}_{2}$ and the dashed lines represent the upper and lower 95\% limits of agreement. 


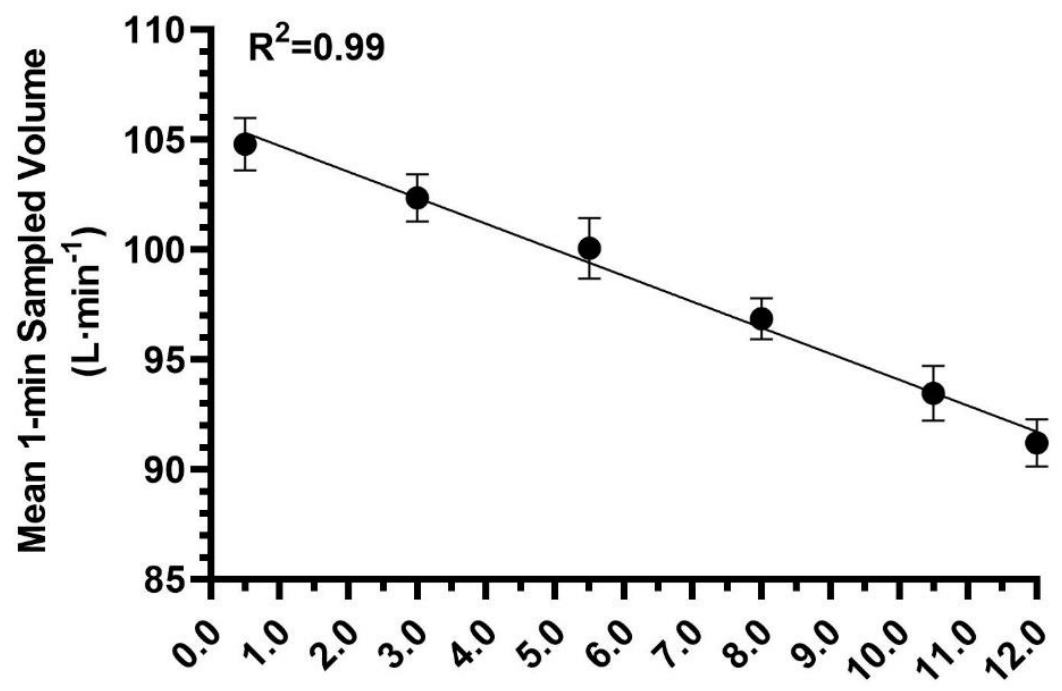

Altitude Setting

570 Figure 3. Hypoxico mean 1-min volume at altitude settings of 0.5, 3.0, 5.5, 8.0, 10.5 571 and 12.0 with a linear regression line (solid black line). Error bars represent $\pm 1 S D$. 